\title{
Toward Strong Field Mode-Selective Chemistry
}

\author{
T. C. Weinacht, J. L. White, P. H. Bucksbaum \\ Physics Department, University of Michigan, Ann Arbor, MI 48109-1120
}

\begin{abstract}
We demonstrate a new method for coherent control of bond excitation in the strong field regime. We can selectively excite one or more Raman-active modes in a molecular liquid without the need for externally generated coherent sources separated by the Stokes frequency. The method employs excitation with an intense, ultrafast, shaped pulse in a learning control loop.
\end{abstract}

\section{INTRODUCTION}

We have constructed an automated learning apparatus [1] to explore non-linear vibrational control mechanisms in molecular liquids. By focusing intense, shaped ultrafast laser pulses into our liquid sample and using signatures of stimulated Raman scattering (SRS) and self phase modulation (SPM) in the forward scattered light as feedback, we were able to reshape the laser pulses to achieve desired forward scattered spectra. The measured spectra were used as input for an evolutionary learning procedure based on a genetic algorithm (GA) [2]. This algorithm ran on a computer, which controlled an acousto-optic modulator (AOM) pulse shaper [3]. The algorithm generated new pulse shapes based on the success of previous ones in achieving our predetermined goals of SPM and vibrational excitation.

\section{Experiment}

The shaped pulses originated from a Kerr lens mode-locked (KLM) titanium sapphire laser, which produced $100 \mathrm{fs}$ pulses with about $5 \mathrm{~nJ}$ of energy at a central wavelength of $790 \mathrm{~nm}$. The pulses were temporally dispersed to $150 \mathrm{ps}$ in a single grating expander and amplified to $2 \mathrm{~mJ}$ in a regenerative amplifier at a repetition rate of 10 $\mathrm{Hz}$. The output of the amplifier was split to form two beams. One beam was sent to the pulse shaper, and the other was recompressed for use as a Fourier transform-limited reference for spectral interferometry [4] measurements of the shaped pulses. To compensate for the low efficiency of the pulse shaper (10-15\%), the shaped pulses were reamplified in a low gain multi-pass amplifier. Then they were recompressed to produce

CP525, Multiphoton Processes: ICOMP VIII, $8^{\text {th }}$ International Conference,

edited by L. F. DiMauro, R. R. Freeman, and K. C. Kulander

(C) 2000 American Institute of Physics 1-56396-946-7/00/\$17.00 
shaped pulses with a minimum pulse width of under $150 \mathrm{fs}$, and an energy of about 1 $\mathrm{mJ}$.

The GA started with a collection (population) of random pulse shapes (individuals). The genetic code of each individual was the spectral phase and amplitude sampled at several different wavelengths (thirty samples for phase and six for amplitude). Each individual was evaluated by measuring its ability (fitness) to generate the spectrum that most resembled the target spectrum. New pulse shapes (next generation) were generated by combining elements of successful pulse shapes from the current generation (mating), and by adding a small amount of random phase or amplitude (mutation). We used two point crossover and $5 \%$ mutation in our implementation of the $\mathrm{GA}^{1}$

\section{Results}

To assess of the capabilities of the apparatus, we studied non-linear frequency shifts of intense light propagating through $1 \mathrm{~cm}$ of liquid $\mathrm{CCl}_{4}$. One set of goals was established based on the observation of periodic spectral modulations in the measured transmission spectra when a transform limited pulse entered the cell. These modulations are typical spectral features for pulses that have undergone SPM under the influence of an intensity-dependent index of refraction. We used the GA to enhance these modulations. We were able to control their frequency and phase by altering the shape of the driving pulse. Figure 1 demonstrates control over these spectral modulations.

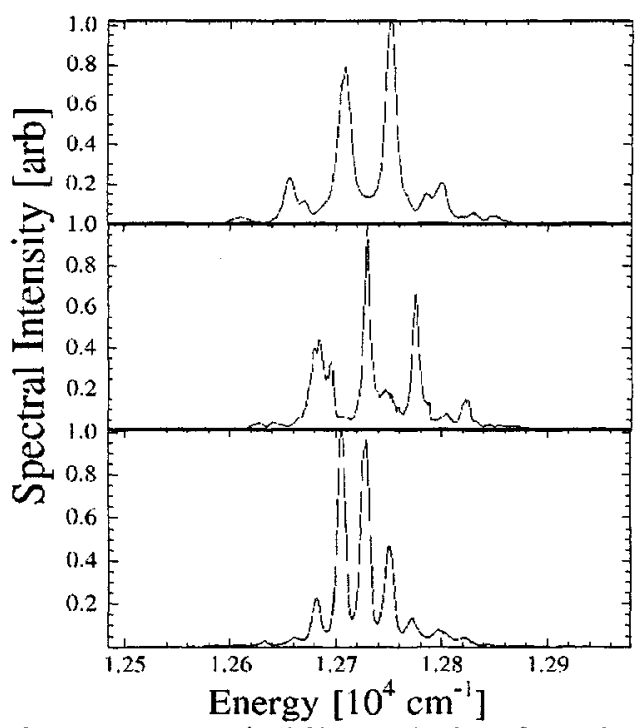

FIGURE 1. SPM spectra in $\mathrm{CCl}_{4}$. Panels show forward scattered spectra for three different pulses optimized to change phase and frequency of spectral modulations.

\footnotetext{
${ }^{1}$ Two point crossover is a scheme for generating two new pulse shapes (children) by randomly selecting two points in the gene string and exchanging the genetic code of two "parent" indviduals between these two points.
} 
Once we had evidence that we were able to control intensity modulations in the spectrum by changing the phase of the shaped pulses through SPM, we attempted to control the interaction between the shaped laser pulses and the vibrational modes of a multi-mode molecule. Using the GA, with the integrated spectral intensity of Raman stokes peaks in the forward scattered light as the basis for our feedback signal, we were able to find pulse shapes that were capable of selectively exciting $\mathrm{C}-\mathrm{H}$ stretch modes in methanol. We could excite both the symmetric and asymmetric stretch modes, or either one individually by changing the shape of the driving pulse. The stokes shifts for the asymmetric and symmetric modes are $2942 \mathrm{~cm}^{-1}$ and $2835 \mathrm{~cm}^{-1}$ respectively.

Figure 2 shows evidence for selective excitation of the symmetric and asymmetric stretch modes in methanol. The forward scattered spectra for three separate incident pulses are shown. The solid line shows the spectrum for a pulse which was shaped to optimize excitation of the asymmetric stretch mode, and the dashed shows the spectrum for a pulse optimized to excite the symmetric stretch mode. The curve lying almost along the $x$-axis (dotted line) is the forward scattered spectrum for an unshaped pulse.

\section{Selective Stokes Peaks in Methanol}

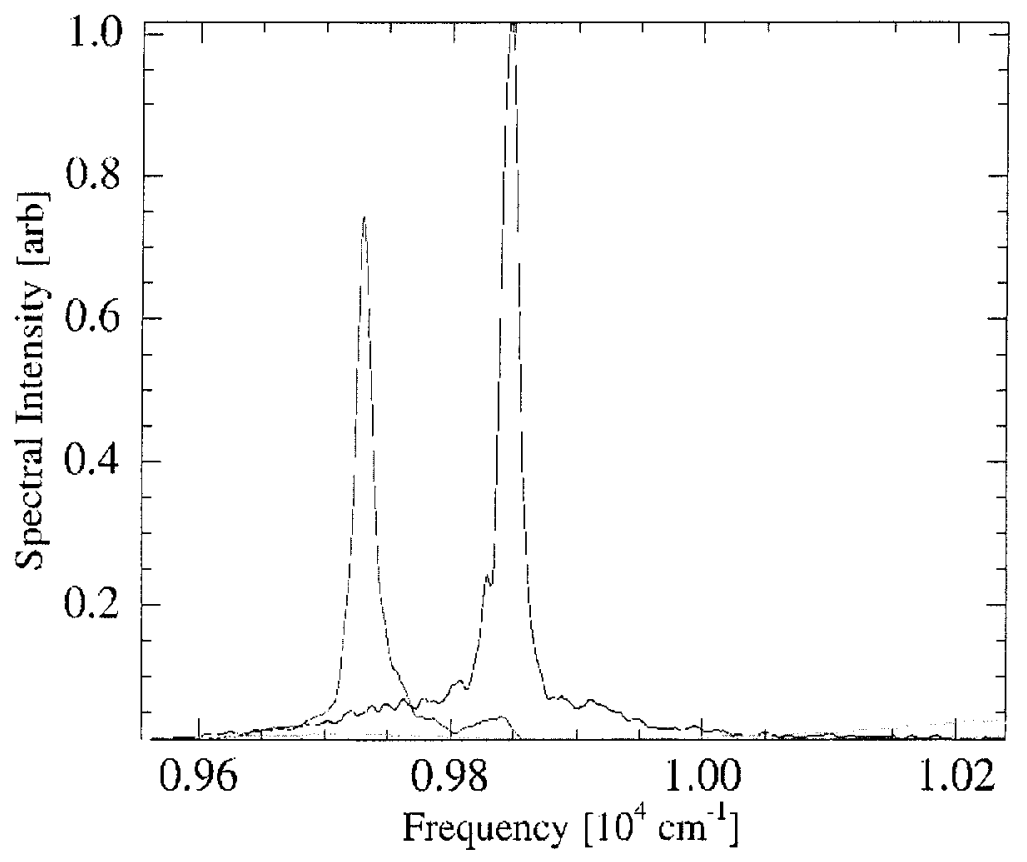

FIGURE 2. Selective Stokes peaks in the forward scattered light with a methanol sample. The driving laser pulse had a central frequency of $12,700 \mathrm{~cm}^{-1}$. 


\section{Discussion}

The Stokes shift for these two modes is large compared to the bandwidth of the driving laser pulse. Therefore, the Raman excitation was non-impulsive since by Fourier's theorem the laser pulse duration was long compared to the vibrational period of either stretch mode. In the non-impulsive regime, one cannot control mode selectivity through direct seeding of the stokes radiation with the laser light.

We have considered two different mechanisms that could account for selective excitation of the $\mathrm{C}-\mathrm{H}$ stretch modes in methanol. One is coupling between molecular polarizability and vibration. The $\mathrm{CCl}_{4}$ experiment shows that light generated by SPM (through a non-linear molecular polarizability) is very sensitive to the input pulse shape. Thus the molecular polarizability might effectively control vibrational excitation by providing seed light for SRS into one or more Raman-active modes.

A second possible mechanism that could account for the selective excitation is quasi-impulsive coupling between the two modes. Although the coupling between different levels of each mode is definitely non-impulsive, the splitting between the two first order stokes shifts is less than the laser bandwidth, so that impulse scattering between them is possible. In the time domain, this is equivalent to saying that the laser pulse is long compared to each normal mode frequency but short compared to the beat note between the two.

We have begun to explore the implications of these two different models of modeselection in our system. We first decided to determine whether the effect is an interor intramolecular effect. We performed an experiment in which we attempted to select between two modes that were in different molecules. The shaped pulses were focussed into a cell that contained an even mixture of benzene $\left(\mathrm{C}_{6} \mathrm{H}_{6}\right)$ and deuterated benzene $\left(\mathrm{C}_{6} \mathrm{D}_{6}\right)$. We attempted to excite the symmetric breathing mode of either the $\mathrm{C}_{6} \mathrm{H}_{6}\left(v=992 \mathrm{~cm}^{-1}\right)$ or the $\mathrm{C}_{6} \mathrm{D}_{6}\left(v=947 \mathrm{~cm}^{-1}\right)$. As with the methanol experiment, both modes have periods that are very short compared to the incident laser pulse, but the beat period between the two modes is long compared to the laser pulse. Figure 3a shows the Raman spectra for pulses focussed into $\mathrm{C}_{6} \mathrm{H}_{6}$ (solid curve) and $\mathrm{C}_{6} \mathrm{D}_{6}$ (dotted curve) alone. Figure $3 \mathrm{~b}$ shows that when a 50/50 mixture was excited, and the genetic algorithm was programmed to excite one or the other mode alone, it was only possible to excite the $\mathrm{C}_{6} \mathrm{H}_{6}$, and not the $\mathrm{C}_{6} \mathrm{D}_{6}$ which has a slightly lower cross-section. Our inability to separately control these two modes, which are spectrally similar to the two modes in methanol, is suggestive. We hypothesize that mode selection may depend in some way on intramolecular processes, since when the light is forced to work with a mixture of different modes on different molecules, the GA is less effective. Perhaps rapid energy redistribution between modes is required. If the benzene control experiment had worked in the mixture, allowing us to choose whether to excite the $\mathrm{C}_{6} \mathrm{H}_{6}$ or the $\mathrm{C}_{6} \mathrm{D}_{6}$, then the mechanism for control could not be completely explained in terms of an internal energy redistribution inside of each individual molecule. 


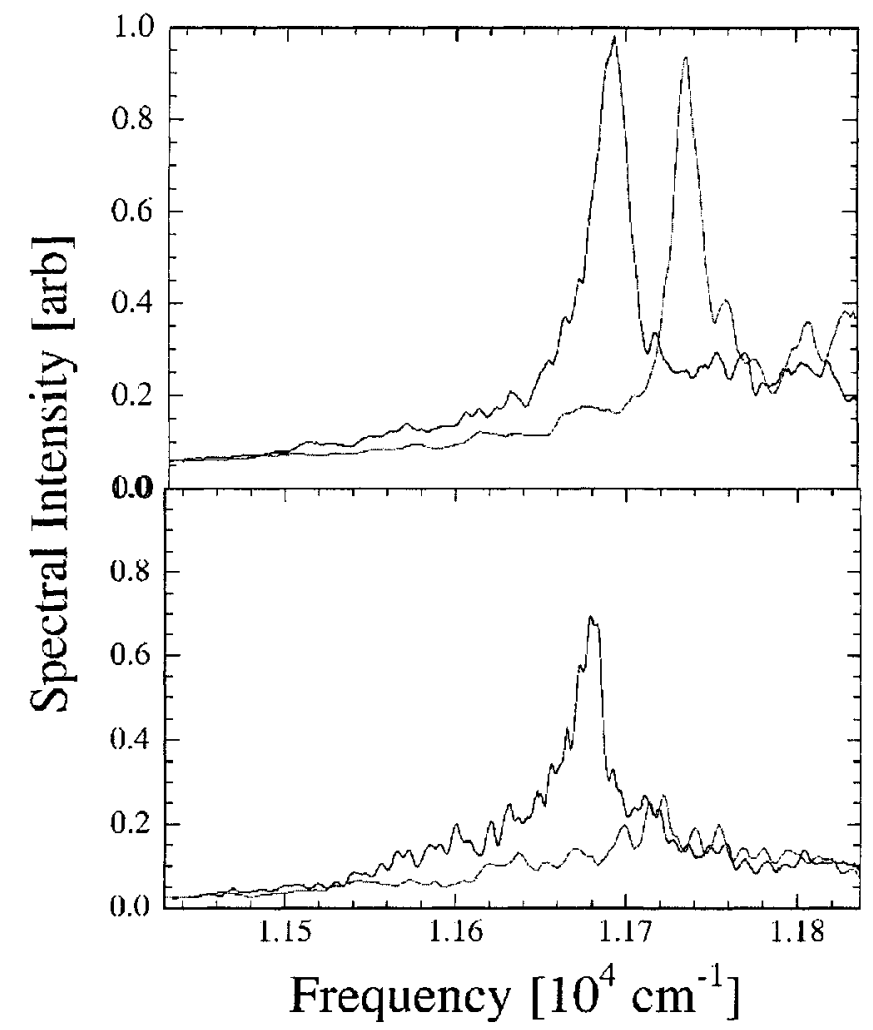

FIGURE 3. (a) Stimulated Raman spectra for $\mathrm{C}_{6} \mathrm{H}_{6}$ and $\mathrm{C}_{6} \mathrm{D}_{6}$ separately. (b) Stimulated spectra for a $50 / 50$ mixture.

Further insight into the selective excitation mechanism can be gained through examination of the shaped pulses that effected the control. Not all genetic algorithm trials converged to the same pulse shapes and we have not completed enough trials to draw final conclusions, but there are some features in the solutions thus far that merit attention.

Figure 4 shows the time and frequency content of optimized electromagnetic field pulses in Husimi representations [4]. These are the converged pulse shapes optimized for exciting the two $\mathrm{C}-\mathrm{H}$ stretch modes in methanol. The Husimi distribution is a common representation for quantum mechanical wavefunctions, but it is also quite useful for these laser spectrograms. These Husimi distributions, $Q(\tau, v)$, for the optimal pulses, were calculated by transforming the optical field $\mathrm{E}(v)$ according to:

$$
\begin{aligned}
& Q(t, v)=\int d v^{\prime} \int d t^{\prime} S\left(t^{\prime}, v^{\prime}\right) e^{-\left(t-t^{\prime}\right)^{2}-\left(v-v^{\prime}\right)^{2}} \\
& S(t, v)=\int E\left(v+v^{\prime}\right) E^{*}\left(v-v^{\prime}\right) e^{-4 \pi v^{\prime} t} d v^{\prime}
\end{aligned}
$$

The electric field $\mathrm{E}(v)$ was determined by combining the converged power spectra with the phase shifts applied to the AOM, which was calibrated by spectral interfer- 
ometry with the unshaped reference. Figure 4 a shows the Husimi distribution for a pulse optimized to excite the asymmetric stretch mode and $4 \mathrm{~b}$ shows an optimal pulse for excitation of the symmetric stretch mode.
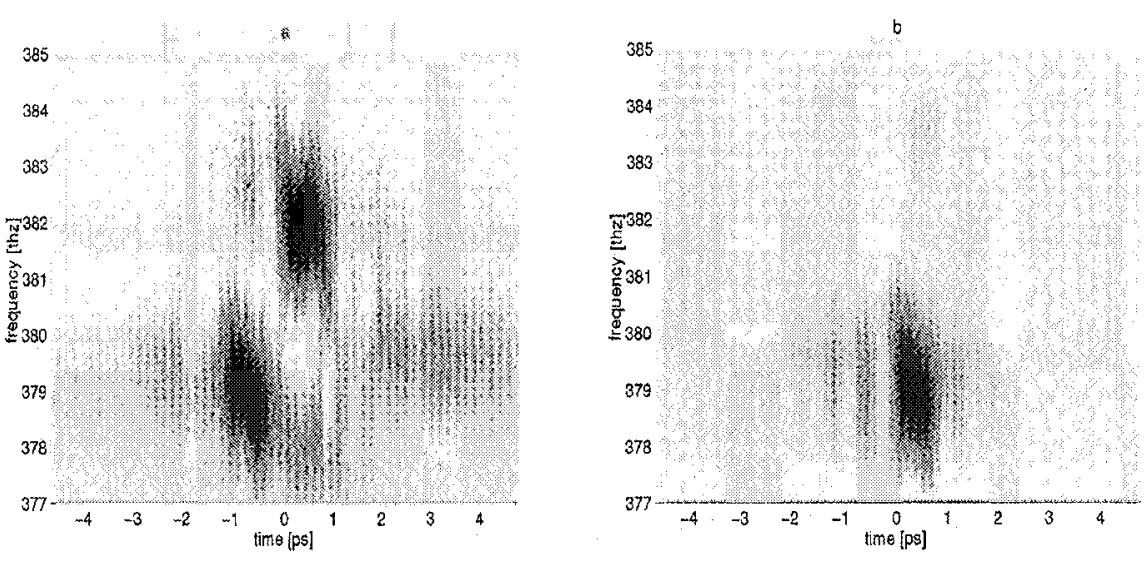

FIGURE 4. Husimi distributions for shaped pulses optimized for driving the symmetric (on the left) and asymmetric (on the right) stretch modes in methanol.

One of the most interesting features is the fact that the optimal pulse for exciting the symmetric stretch has only one spectral lobe whereas the asymmetric stretch has two temporally and spectrally separated spectral lobes. These pulse shapes are consistent with a quasi-impulsive scattering mechanism. If the population in the two levels can be controlled through a quasi-impulsive coupling, then spectral components that are separated by the mode splitting are especially important for control. This could explain the absence of spectral intensity at the center of the spectrum. The separation of the two spectral components in the pulse optimized for excitation of the asymmetric stretch is the same as the energy separation between the two modes in the molecule.

Although the benzene experiment and the optimized pulse shapes are both consistent with a quasi-impulsive picture for the mode selective mechanism, they do not unambiguously indicate that quasi-impulsive coupling is the dominant mechanism. There are several technical details that complicate the data collection and we are currently working on different ways of probing the excited molecular states to uncover the mode selection mechanism.

Raman scattering in conjunction with other non-linear but non-resonant processes as a control technique is an attractive approach because of its generality. The technique is inherently non-resonant, so it can be applied to a wide range of molecules with a wide range of light sources. Transparent molecular liquids with low group velocity dispersion and non-degenerate vibrations are ideal candidates for this type of control. If quasi-impulsive scattering is responsible for the control, then increasing the laser bandwidth is an essential step in generalizing the technique to include many more molecular modes. However, the bandwidth requirements for a quasi-impulsive 
scattering are in general less than for impulsive scattering since quasi-impulsive coupling requires bandwidths on the order of frequency differences between modes whereas impulsive coupling requires bandwidths on the order of the mode frequencies themselves.

Future goals include looking at vibrational overtones and combining closely spaced modes to activate single bonds in molecules. This might allow us to control reactions in bimolecular solutions that depend sensitively on bond lengths for activation.

\section{ACKNOWLEDGMENTS}

The authors would like to acknowledge very helpful and informative discussions with Roberto Merlin, Noemi Mirkin, Ronnie Kosloff, Sandy Ruhman, Roseanne Sension, Ian Walmsley, Douglass Schumacher and Herschel Rabitz. This work was supported by the NSF under grant number 9414335 .

\section{REFERENCES}

1. Judson, R. S., and Rabitz, H. Phys, Rev. Letters 68, 1500 (1992)

2. Davis, L. Handbook of Genetic Algorithms (1991)

2. Tull, J. X.., Dugan, M. A., Warren, W. S. Adv. Opt. Mag. Resonance 1 (1990)

3. Froehy, C., LaCourt, A., and Vienot, J. C. Journal of Optics 4, 183 (1973); Piasecki, J., Colombeau, Vanpouille, M., Froehy, C., and Arnaud, J. A. Applied Optics 193749 (1980)

4. Paye, J. IEEE Journal of Quantum Electronics 28, 2262-2273 (1992) 